\title{
Moving Toward Multicenter Therapeutic Trials in Amyotrophic Lateral Sclerosis: Feasibility of Data Pooling Using Different Translocator Protein PET Radioligands
}

\author{
Donatienne Van Weehaeghe*1, Suma Babu*2, Joke De Vocht ${ }^{3}$, Nicole R. Zürcher ${ }^{4}$, Sheena Chew $^{2}$, Chieh-En J. Tseng ${ }^{4}$, \\ Marco L. Loggia ${ }^{4}$, Michel Koole ${ }^{1}$, Ahmadreza Rezaei ${ }^{1}$, Georg Schramm ${ }^{1}$, Philip Van Damme ${ }^{3}$, Jacob M. Hooker ${ }^{4}$, \\ Koen Van Laere*1, and Nazem Atassi*2 \\ ${ }^{I}$ Nuclear Medicine Subdivision, Department of Imaging and Pathology, University Hospital Leuven, Leuven, Belgium; ${ }^{2}$ Department of \\ Neurology, Sean M. Healey and AMG Center for ALS, Massachusetts General Hospital, Harvard Medical School, Boston, \\ Massachusetts; ${ }^{3}$ Department of Neurology, University Hospital Leuven, and Laboratory of Neurobiology, Center for Brain and \\ Disease Research, VIB, Leuven, Belgium; and ${ }^{4}$ Athinoula A. Martinos Center for Biomedical Imaging, Massachusetts General \\ Hospital, Harvard Medical School, Charlestown, Massachusetts
}

Neuroinflammation has been implicated in amyotrophic lateral sclerosis (ALS) and can be visualized using translocator protein (TSPO) radioligands. To become a reliable pharmacodynamic biomarker for ALS multicenter trials, TSPO radioligands have some challenges to overcome. We aimed to investigate whether multicenter data pooling of different TSPO tracers $\left({ }^{11} \mathrm{C}\right.$-PBR28 and ${ }^{18} \mathrm{~F}$-DPA714) is feasible, after validation of an established ${ }^{11} \mathrm{C}$-PBR28 PET pseudo reference analysis technique for ${ }^{18} \mathrm{~F}-\mathrm{DPA} 714$. Methods: Seven ALS patients from Belgium ( $58.9 \pm 6.7$ y old, 5 men and 2 women), 8 healthy volunteers from Belgium $(52.1 \pm 15.2$ y old, 3 men and 5 women), 7 ALS patients from the United States ( $53.4 \pm 9.8$ y old, 5 men and 2 women), and 7 healthy volunteers from the United States (54.6 \pm 9.6 y old, 4 men and 3 women) from a previously published study underwent dynamic ${ }^{18} \mathrm{~F}-\mathrm{DPA} 714$ (Leuven, Belgium) or ${ }^{11} \mathrm{C}-$ PBR28 (Boston, Massachusetts) PET/MRI. For ${ }^{18}$ F-DPA714, maps of total volume of distribution $\left(\mathrm{V}_{\mathrm{T}}\right)$ were compared with SUV ratio (SUVR) images from 40 to 60 min after injection (SUVR $_{40-60}$ ) calculated using the pseudo reference regions cerebellum, occipital cortex, and whole brain (WB) without ventricles. For ${ }^{11} \mathrm{C}-\mathrm{PBR} 28$, SUVR images from 60 to 90 min after injection using the WB without ventricles were calculated. Results: In line with previous studies, increased ${ }^{18} \mathrm{~F}$-DPA714 uptake $(17.0 \% \pm 5.6 \%)$ in primary motor cortices was observed in ALS subjects, as measured by both $V_{T}$ and SUVR ${ }_{40-60}$ approaches. The highest sensitivity was found for SUVR calculated using the WB without ventricles (average cluster, $21.6 \% \pm 0.1 \%) .{ }^{18} \mathrm{~F}-\mathrm{DPA} 714 \mathrm{~V}_{\mathrm{T}}$ ratio was highly correlated with the SUVR $_{40-60}(r>0.8, P<0.001)$. A similar pattern of increased uptake (average cluster, $20.5 \% \pm 0.5 \%$ ) in the primary motor cortices was observed in ALS subjects for ${ }^{11} \mathrm{C}$-PBR28 SUVR calculated using the WB without ventricles. Analysis of the ${ }^{18} \mathrm{~F}-\mathrm{DPA} 714$ and ${ }^{11} \mathrm{C}-\mathrm{PBR} 28$ data together resulted in a more extensive pattern of significantly increased glial activation bilaterally in the primary motor cortices. Conclusion: The same pseudo reference region analysis technique for ${ }^{11} \mathrm{C}$-PBR28 PET can be extended toward ${ }^{18} \mathrm{~F}-\mathrm{DPA} 714$ PET. Therefore, in ALS, standardized analysis across these 2 tracers enables pooling of TSPO PET data across multiple

Received Dec. 15, 2019; revision accepted Mar. 5, 2020.

For correspondence or reprints contact: Donatienne Van Weehaeghe, University Hospital Leuven, Herestraat 49, 3000 Leuven, Belgium.

E-mail: donatienne.vanweehaeghe@uzleuven.be

${ }^{*}$ Contributed equally to this work.

Published online Mar. 13, 2020.

COPYRIGHT (c) 2020 by the Society of Nuclear Medicine and Molecular Imaging. centers and increases the power of TSPO as a biomarker for future therapeutic trials.

Key Words: amyotrophic lateral sclerosis; translocator protein; multicenter; ${ }^{11} \mathrm{C}$-PBR28; ${ }^{18} \mathrm{~F}-\mathrm{DPA} 714$

J Nucl Med 2020; 61:1621-1627

DOI: 10.2967/jnumed.119.241059

A myotrophic lateral sclerosis (ALS) is a rare and fatal neurodegenerative disease that causes progressive weakness, respiratory failure, and eventual death. Presently, there is no cure for ALS, thus putting ALS drug development front and center for any ALS research. Therapeutic drug development in ALS has unique challenges to overcome, including outcome measures that are not tailored to the phenotypic variability of the disease, heterogeneity in disease and mechanisms, and lack of molecular biomarkers of disease mechanisms (1). There is an urgent unmet need to develop mechanistic molecular biomarkers in ALS that can be used across multiple sites and serve as early and reliable readouts of treatment efficacy.

Neuroinflammation, reflected by glial activation, is implicated in the pathophysiology of ALS (2,3). With recent advancements in imaging technology, glial activation is now identifiable and measurable in vivo using translocator protein (TSPO) PET (4). Unfortunately, genotyping before scanning is necessary because of the existence of a polymorphism affecting binding affinity and dividing the population into high-affinity binders, medium-affinity binders, and low-affinity binders (5).

TSPO expression is notably increased in activated microglia and reactive astrocytes, which are essential cell types implicated in central nervous system inflammation in ALS. ${ }^{11} \mathrm{C}-\mathrm{PBR} 28(\mathrm{~N}-((2-$ (methoxy- $\left.{ }^{11} \mathrm{C}\right)$ phenyl)methyl)- $N$-(4-phenoxy-3-pyridinyl)-acetamide), an acetamide, and ${ }^{18} \mathrm{~F}-\mathrm{DPA} 714$ ( ${ }^{18} \mathrm{~F}$-labeled $\mathrm{N}, \mathrm{N}$-diethyl-2-[4-(2fluoroethoxy)phenyl]-5,7-dimethylpyrazolo[1,5-a]pyrimidine-3acetamide), a pyrazolopyrimidine, are second-generation TSPO radioligands with a similar specificity and cortical distribution, and they both have a higher bioavailability in brain tissue than do first-generation and other second-generation TSPO radioligands 
in healthy volunteers (HVs) (6). Hence, ${ }^{11} \mathrm{C}-\mathrm{PBR} 28$ and ${ }^{18} \mathrm{~F}-\mathrm{DPA} 714$ are being developed in the United States and Europe, respectively, as promising candidate biomarkers for ALS and other central neuroinflammatory disorders. Evidence from different cohorts in the United States and Europe have independently showed increased uptake of ${ }^{11} \mathrm{C}$-PBR28 and ${ }^{18} \mathrm{~F}$-DPA714, predominantly in the primary motor and premotor cortices in ALS (7-12). Moreover, glial activation measured by ${ }^{11} \mathrm{C}-\mathrm{PBR} 28$ uptake in the primary motor cortices correlated with clinical parameters of functional decline such as the revised ALS functional rating scale and with structural and metabolic measures of neurodegeneration and axon loss such as motor cortical thickness, fractional anisotropy, and spectroscopy changes (myoinositol-to-creatinine and $\mathrm{N}$-acetyl aspartate-to-creatinine ratios) (7-9,11).

Full dynamic modeling using arterial blood sampling during scanning is the gold standard for quantifying tissue uptake of PET tracers (defined as total volume of distribution $\left[\mathrm{V}_{\mathrm{T}}\right]$ ). However, Albrecht et al. demonstrated that a pseudo reference analysis technique can be used for ${ }^{11} \mathrm{C}$-PBR28 PET analysis in ALS patients, with results similar to those for full dynamic modeling (13). The pseudo reference technique defines relative PET tracer uptake in areas of interest, compared with a regional signal in the pseudo reference region (called SUV ratio, or SUVR). This technique reduces patient burden, scan time, and the discomfort of undergoing arterial line placement, compared with the dynamic modeling technique. Furthermore, this technique might enable multicenter pooling of different TSPO radioligands, as it partially corrects for different nuisance variables such as TSPO binding affinity (genotype) and PET camera characteristics. This technique has shown comparable sensitivity in detecting group differences in $\mathrm{V}_{\mathrm{T}}$ in another neurodegenerative disease (14).

In this study, we aimed to validate the pseudo reference analysis technique developed for ${ }^{11} \mathrm{C}-\mathrm{PBR} 28$ using the same automated pipeline for images obtained with a different PET tracer, ${ }^{18}$ F-DPA714, and to define the pseudo reference region with the highest sensitivity. We also investigated the validity of pooling 2 different ALS and HV cohorts using 2 different TSPO tracers $\left({ }^{11} \mathrm{C}-\mathrm{PBR} 28\right.$ and ${ }^{18} \mathrm{~F}$ DPA714) and 2 different scanners.

\section{MATERIALS AND METHODS}

\section{Demographics}

Demographics are illustrated in Table 1. Genotyping of the rs6971 polymorphism was performed on venous samples in all participants to determine TSPO binding affinity (5).

${ }^{18} \mathrm{~F}$-DPA714 (Belgium). Eight HVs $(52.1 \pm 15.2$ y old, 3 men and 5 women, 7 medium-affinity binders and 1 low-affinity binder) were recruited using departmental websites, flyers, and advertisement boards ("Ad Valvas"). They had no history of major internal, neurologic, or psychiatric diseases and no first-grade relatives with ALS or related diseases (primary lateral sclerosis, progressive muscular atrophy, or frontotemporal dementia).

Seven ALS participants $(58.9 \pm 6.7$ y old, 5 men and 2 women, 3 high-affinity binders, 3 medium-affinity binders, and 1 low-affinity binder) were recruited by an experienced neurologist from the neuromuscular department of the tertiary University Clinic UZ Leuven. All participants were diagnosed with sporadic ALS and had symptoms for less than $1 \mathrm{y}$. Exclusion criteria included chronic anti-inflammatory drugs (except inhalation corticoids) or immune modulatory agents.

${ }^{11} C-P B R 28$ (the United States). Seven HVs (54.6 \pm 9.6 y old, 4 men and 3 women, 5 high-affinity binders and 2 medium-affinity binders) and 7 ALS participants (53.4 \pm 9.8 y old, 5 men and 2 women, 4 high-affinity binders and 3 medium-affinity binders) from the study of Zürcher et al. (7) were selected to match the Belgian participants in sex and age. Although the U.S. ALS subjects with the shortest disease duration were selected, disease duration $(6.3 \pm 2.7 \mathrm{y}$ for the Belgian ALS group vs. $14.7 \pm 4.7 \mathrm{y}$ for the U.S. ALS group) and revised ALS functional rating scale (43.4 \pm 1.9 for the Belgian ALS group vs. $38.1 \pm 4.4$ for the U.S. ALS group) were significantly different from those of the Belgian ALS group (Table 1).

The study was performed in accordance with the World Medical Association Declaration of Helsinki and approved by the local University Ethics Committees and Institutional Review Boards, respectively. Written informed consent was obtained from all the participants before study participation.

\section{Data Acquisition and Processing}

${ }^{18}$ F-DPA714 (Belgium). Participants underwent 60-min dynamic PET/MRI on a GE Healthcare Signa 3-T PET/MR system in list mode. The mean injected dose was $144 \pm 20 \mathrm{MBq}$. During the PET acquisition, several MRI acquisitions took place. For this study, the sequences we used were 3-dimensional volumetric T1-weighted Bravo (a proprietary GE Healthcare sequence: plane, oblique; echo time, $3.2 \mathrm{~ms}$; repetition time, $8.5 \mathrm{~ms}$; inversion time, $450 \mathrm{~ms}$; flip angle, 12; receiver bandwidth, 31.2; number of excitations, 1) and 3-dimensional T2-weighted Cube (a proprietary GE Healthcare sequence: plane, oblique; echo time, $136 \mathrm{~ms}$; number of echoes, 1; echo train length, 190; repetition time, 8,500 ms; inversion time, $50 \mathrm{~ms}$; receiver bandwidth, 31.25 ; number of excitations, 1) fluid-attenuated inversion recovery. Multichannel segmentation (in Statistical Parametric Mapping software, version 12) was performed to calculate subject-specific tissue probability maps (gray matter, white matter, and cerebrospinal fluid) using Bravo and fluid-attenuated inversion recovery.

List-mode PET data were unlisted into dynamic frames of $4 \times 15 \mathrm{~s}$, $4 \times 60 \mathrm{~s}, 2 \times 150 \mathrm{~s}$, and $10 \times 300 \mathrm{~s}$ and reconstructed with orderedsubsets expectation maximization (4 iterations, 28 subsets). Reconstructions were corrected for scatter, dead time, and time-of-flight offset (15), with isotropic gaussian postsmoothing (full width half maximum, $4.5 \mathrm{~mm})$. A zero-echo-time sequence was used for attenuation correction (16). Motion correction by means of rigid coregistration of every frame to the averaged first 10 frames was performed if necessary. Manual arterial sampling was performed at gradually increasing intervals from $10 \mathrm{~s}$ after injection to the end of the scan. In total, 5 arterial blood samples were used to create the metabolite-corrected arterial input function (5, 10, 20, 40, and $60 \mathrm{~min}$ after injection). Plasma and blood activity curves were constructed, and plasma-to-whole-blood ratios were calculated. The percentage of intact tracer was calculated using a plasma radiometabolite analysis. Blood and plasma activity were fitted with a 3-exponential model curve, whereas plasma radiometabolite data were fitted with the Hill function.

Spatial normalization (to Montreal Neurologic Institute space) was performed using both Bravo and 3-tissue-probability-map transformation (PMOD, version 3.9; PMOD Technologies). Voxel-based $\mathrm{V}_{\mathrm{T}}$ images were created using Logan graphical analysis (PMOD, version 3.9) with blood volume fixed to $5 \%$ and $t^{*}$ to $31 \mathrm{~min}$ (17). SUVR images from 40 to $60 \mathrm{~min}$ after injection $\left(\mathrm{SUVR}_{40-60}\right)$ were created, using cerebellum, occipital cortex, and whole brain (WB) without ventricles as pseudo reference regions. WB without ventricles consists of an individual gray and white matter mask without the ventricles.

${ }^{11} \mathrm{C}$-PBR28 (the United States). Data were acquired on a Siemens 3-T Tim Trio PET/MR scanner. The T1-weighted magnetization-prepared rapid gradient-echo sequence was used for anatomic localization, spatial normalization, and attenuation correction (18). Details on the PET acquisition were previously published (7). For ${ }^{11} \mathrm{C}-\mathrm{PBR} 28$, SUVR images from 60 to 90 min after injection $\left(\mathrm{SUVR}_{60-90}\right.$ ), as done previously (79,11,13), and SUVR $40-60$ images to match the ${ }^{18} \mathrm{~F}$-DPA714 time window were created using WB without ventricles as the pseudo reference region. 
TABLE 1

Demographics

\begin{tabular}{|c|c|c|c|c|c|}
\hline \multirow[b]{2}{*}{ Demographic } & \multicolumn{2}{|l|}{ Belgium } & \multicolumn{2}{|c|}{ United States } & \multirow[b]{2}{*}{$P$} \\
\hline & ALS & $\mathrm{HV}$ & ALS & $\mathrm{HV}$ & \\
\hline Age $(y)$ & $58.9 \pm 6.7$ & $52.1 \pm 15.2$ & $53.4 \pm 9.8$ & $54.6 \pm 9.6$ & 0.72 \\
\hline $\operatorname{Sex}(n)$ & & & & & 0.49 \\
\hline Men & 5 & 3 & 5 & 4 & \\
\hline Women & 2 & 5 & 2 & 3 & \\
\hline TSPO binding affinity & & & & & 0.12 \\
\hline High-affinity binder & 3 & 0 & 4 & 5 & \\
\hline Medium-affinity binder & 3 & 7 & 3 & 2 & \\
\hline Low-affinity binder & 1 & 1 & 0 & 0 & \\
\hline ALSFRS-R & $43.4 \pm 1.9$ & & $38.1 \pm 4.4$ & & 0.02 \\
\hline VC (\% predicted) & Forced VC: $106.7 \pm 25.0$ & & Slow VC: $84.3 \pm 16.1$ & & 0.09 \\
\hline Disease duration (mo) & $6.3 \pm 2.8$ & & $14.7 \pm 4.7$ & & 0.003 \\
\hline
\end{tabular}

\section{Data Analysis}

Region-based partial-volume correction (PVC) was applied to all ${ }^{11} \mathrm{C}$ PBR28 and ${ }^{18}$ F-DPA714 scans (FreeSurfer, version 6.0; Laboratory for Computational Neuroimaging), and the Desikan-Killiany atlas was used for region delineation. General statistics were performed in SPSS (version 25.0). The significance threshold was set at a $P$ value of less than 0.05 .

For ${ }^{18} \mathrm{~F}-\mathrm{DPA} 714$, the mean bilateral ratios of uptake in the primary motor cortices (defined as the average of precentral and paracentral gray matter gyri) to uptake in the occipital cortex, cerebellum, and WB without ventricles were calculated, for both $\mathrm{V}_{\mathrm{T}}$ and $\mathrm{SUVR}_{40-60}$ analyses, to correct for genotype. Normality was tested using the Shapiro-Wilk test. Pearson correlation was used to correlate $V_{T}$ ratio with SUVR $40-60$. Student $t$ tests were performed to investigate $\mathrm{V}_{\mathrm{T}}$ and SUVR $_{40-60}$ differences between ALS subjects and HVs.

To assess the validity of combining the data from cohorts scanned by the 2 different tracers, a full factorial analysis was performed followed by a pooled $t$ test for validation using both tracers to compare group differences (ALS vs. HV). A full factorial analysis allows investigation of how regional brain uptake was influenced by tracer and by disease effects. Additionally, the Glass calculation of effect size [(ALS mean - HV mean)/HV SD] was used to investigate the ability of both tracers to differentiate between ALS subjects and HVs. Full factorial analysis was repeated using sex, age, and TSPO binding status as covariates.

Surface-based comparisons of ALS SUVR images to HV SUVR images were performed after gaussian smoothing $(6 \mathrm{~mm})$ using the tracer as the covariate (projection factor, 0.5; uncorrected $P<0.05$, cluster $P$ corrected for familywise error $<0.05$ ) (FreeSurfer, version 6.0).

\section{RESULTS}

\section{Optimal Pseudo Reference Region for ${ }^{18} \mathrm{~F}$-DPA714}

Significantly increased mean ${ }^{18} \mathrm{~F}-\mathrm{DPA} 714$ uptake was observed bilaterally in the primary motor cortices of ALS subjects versus HVs $(17.0 \% \pm 5.6 \%$ after PVC). This finding was observed using both dynamic modeling and pseudo reference analysis techniques and was significant for $\mathrm{V}_{\mathrm{T}}$ ratio using occipital cortex $(P$ $=0.026), \mathrm{V}_{\mathrm{T}}$ ratio using $\mathrm{WB}$ without ventricles $(P=0.037)$,

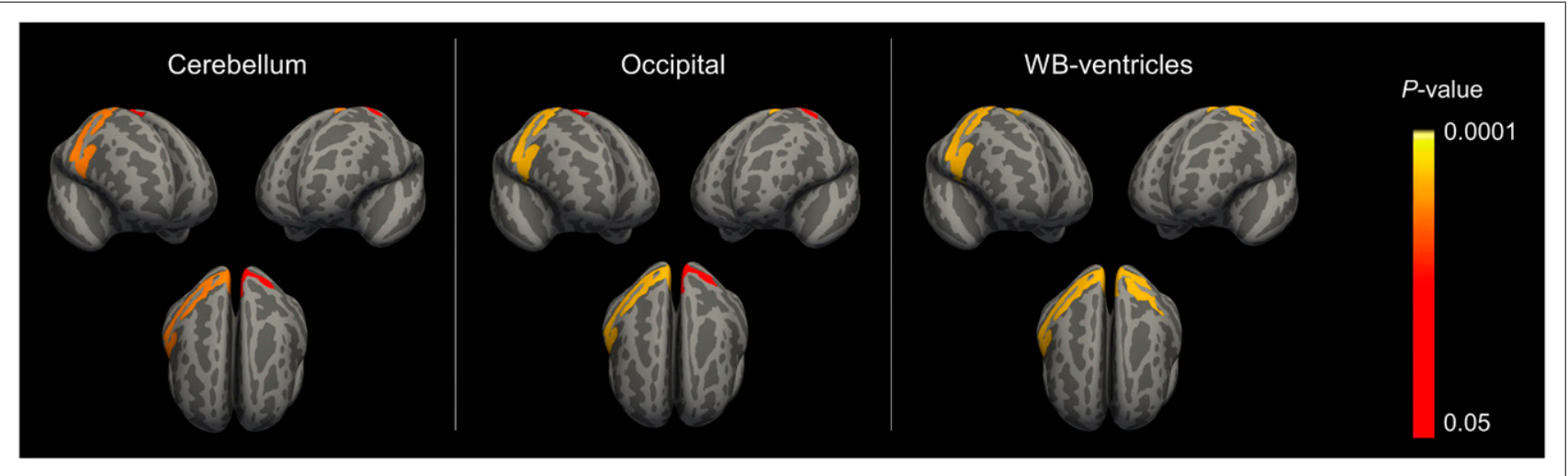

FIGURE 1. Surface-based analysis for ${ }^{18} \mathrm{~F}-\mathrm{DPA} 714$ in ALS group compared with HV group on SUVR $40-60$ with 0.5 projection factor and 6-mm gaussian smoothing was performed using cerebellum, occipital cortex, and WB without ventricles (WB-ventricles) as pseudo reference regions. 
SUVR $_{40-60}$ using occipital cortex $(P=0.019)$, SUVR $_{40-60}$ using cerebellum $(P=0.018)$, and SUVR $_{40-60}$ using WB without ventricles $(P=0.008)$. If the low-affinity binders are excluded, only SUVR $_{40-60}$ using cerebellum $(P=0.047)$ and SUVR $_{40-60}$ using WB without ventricles remain significant $(P=0.022)$. Surface-based analysis showed increased ${ }^{18}$ F-DPA714 uptake bilaterally in the precentral gyri for all 3 pseudo-reference-region normalizations in ALS subjects compared with HVs. The results were most significant and widespread using WB without ventricles (Fig. 1). Increased mean bilateral ${ }^{18} \mathrm{~F}$-DPA714 uptake in the primary motor cortices, compared with the occipital cortex, cerebellum, and WB without ventricles, was similar between the lowaffinity-binder ALS patients and the medium- and high-affinity binders (Fig. 2). No significant differences in absolute $V_{T}$ in the pre- or paracentral gyri or pseudo reference regions were observed between the ALS and HV groups.

Highly significant correlations were observed between $V_{T}$ ratios and SUVR $40-60$ analyses across all pseudo reference regions: occipital cortex (no PVC: $r=0.968, P<0.001$; PVC: $r=$ 0.811, $P<0.001$ ), cerebellum (no PVC: $r=0.975, P<0.001$; PVC: $r=0.891, P<0.001$ ), and WB without ventricles (no PVC: $r=0.917, P<0.001$; PVC: $r=0.902, P<0.001$ ). Bias was lowest using WB without ventricles. The correlations, with the respective fit lines, are illustrated in Figure 2.

\section{Pooling of ${ }^{18} \mathrm{~F}-\mathrm{DPA} 714$ and ${ }^{11} \mathrm{C}-\mathrm{PBR} 28$ (Using WB Without Ventricles as Pseudo Reference Region, After PVC)}

Full factorial analysis showed significant tracer effects in multiple brain regions. Significant group effects (ALS vs. HV) were observed only in left and right paracentral gyri (partial $\eta^{2}, 0.53$ and 0.42 for left and right, respectively) and the right precentral gyrus (partial $\eta^{2}, 0.15$ ). Partial $\eta^{2}$ is a measure of the variance explaining the effect (tracer or group). The group effect was explained only by the bilateral uptake in the paracentral and right precentral gyrus, and the tracer effect was explained by the differential uptake in multiple brain regions. After including age, sex, and TSPO binding status as covariates, the absolute difference decreased slightly given the more stringent analysis; however, the group effect remained significant in the left and right paracentral gyri and the right precentral gyrus. No significant interaction was observed between tracer and group effect.

Pooled $t$ testing, to validate the full factorial analysis, showed a significantly higher uptake in the right precentral and left and right paracentral gyri (average, $17.8 \% \pm 4.0 \%$ ) in ALS subjects than in HVs. This finding was confirmed in the surface-based analysis, which showed higher TSPO uptake (average cluster increase, $16.0 \% \pm 2.1 \%$ ) in the left and right precentral and right paracentral gyri in ALS subjects than in HVs after pooling of both tracers, using the tracer as the covariate. The effect sizes were 1.3 for left paracentral gyrus, 1.3 for left precentral gyrus, 1.3 for right paracentral gyrus, and 1.2 for right precentral gyrus for ${ }^{18}$ F-DPA714 SUVR ${ }_{40-60}$ and, respectively, 1.2, 0.1, 1.4, and 1.8 for ${ }^{11}$ C-PBR28 SUVR $60-90$ (Table 2).

Similarly, surface-based analysis showed increased uptake in the left and right precentral gyri (average cluster increase, $21.6 \% \pm 0.1 \%$ ) and in the right paracentral and left superior frontal gyri (average cluster increase, $20.5 \% \pm 0.5 \%$ ) using only ${ }^{18} \mathrm{~F}$ DPA714 SUVR $_{40-60}$ or ${ }^{11}$ C-PBR28 SUVR $60-90$, respectively (Fig. 3). Using $\mathrm{SUVR}_{40-60}$ for both ${ }^{18} \mathrm{~F}-\mathrm{DPA} 714$ and ${ }^{11} \mathrm{C}-\mathrm{PBR} 28$ produced similar results (Supplemental Fig. 1; supplemental materials are available at http://jnm.snmjournals.org).

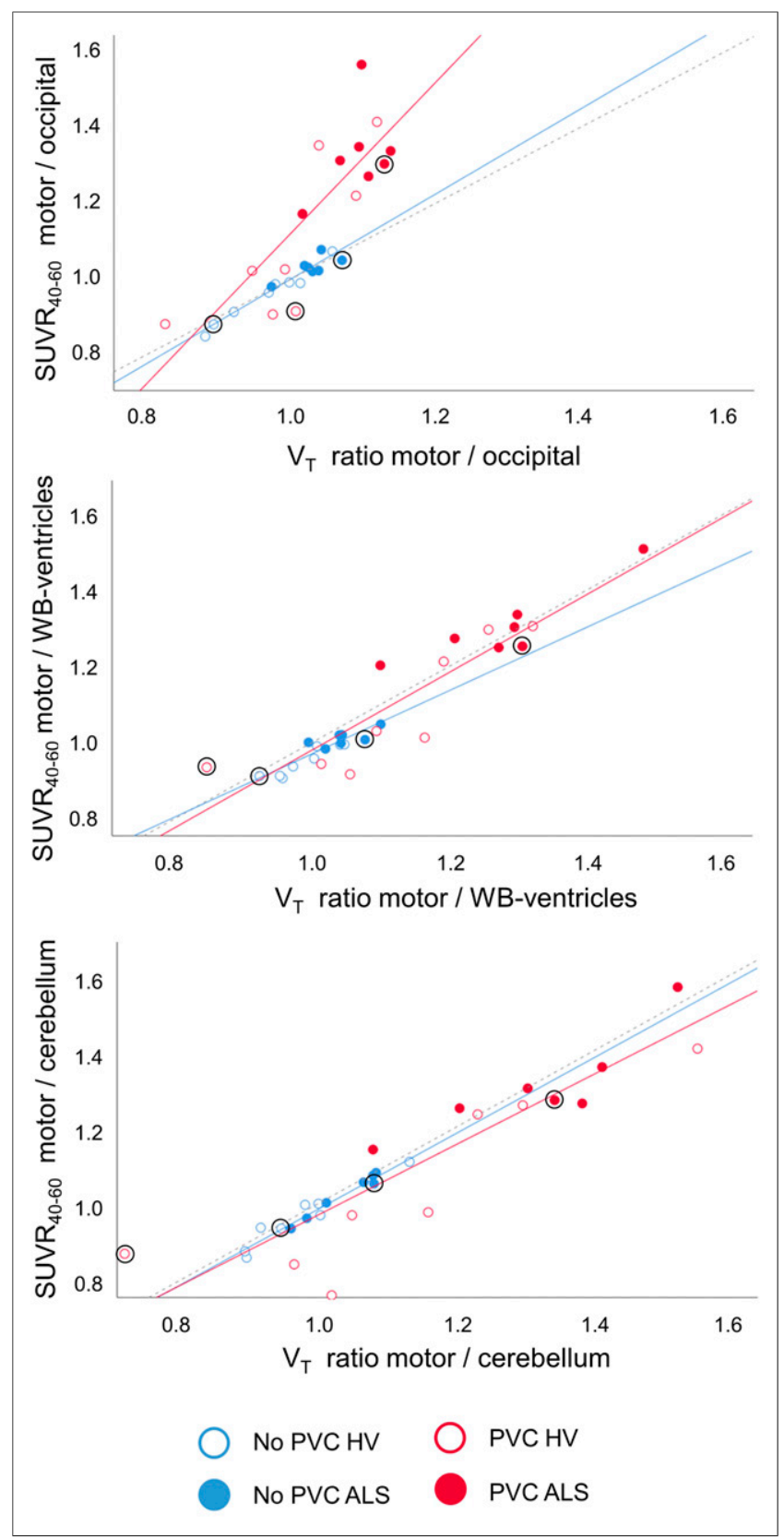

FIGURE 2. Correlations for ${ }^{18} \mathrm{~F}-\mathrm{DPA} 714$ between $\mathrm{V}_{\mathrm{T}}$ and $\mathrm{SUVR} \mathrm{R}_{40-60}$ of left and right primary motor cortices over occipital, cerebellum, and WB without ventricles (WB-ventricles). Blue is non-PVC data; red is after PVC. Solid lines are fitted lines, and dashed line is identity line. Subjects in black circles are low-affinity binders.

\section{DISCUSSION}

In line with previous TSPO PET studies on ALS (7-11,13), this study demonstrated that the uptake of both ${ }^{18} \mathrm{~F}-\mathrm{DPA} 714$ and ${ }^{11} \mathrm{C}$ PBR28 was bilaterally increased in the primary motor cortices in ALS subjects, compared with HVs. Moreover, our results demonstrated that the pseudo reference technique-developed for ${ }^{11} \mathrm{C}$-PBR28 PET-produces results comparable to those of gold standard PET analyses $\left(\mathrm{V}_{\mathrm{T}}\right)$ obtained by kinetic modeling with arterial blood sampling for ${ }^{18} \mathrm{~F}$-DPA714. Notably, the most sensitive (lowest $P$ value and lowest bias) of the pseudo reference regions 
TABLE 2

Mean, SD, and Effect Size for ${ }^{18} \mathrm{~F}-\mathrm{DPA} 714$ SUVR $_{40-60}$ and ${ }^{11} \mathrm{C}-\mathrm{PBR} 28$ SUVR $60-90$ Using WB Without Ventricles as Pseudo Reference Region

\begin{tabular}{|c|c|c|c|c|c|}
\hline Parameter & Agent & Left paracentral & Left precentral & Right paracentral & Right precentral \\
\hline \multirow[t]{2}{*}{ HV mean } & ${ }^{11} \mathrm{C}-\mathrm{PBR} 28$ & 1.34 & 1.39 & 1.25 & 1.22 \\
\hline & ${ }^{18} \mathrm{~F}-\mathrm{DPA} 714$ & 1.04 & 1.22 & 1.02 & 1.07 \\
\hline \multirow[t]{2}{*}{ ALS mean } & ${ }^{11} \mathrm{C}-\mathrm{PBR} 28$ & 1.59 & 1.40 & 1.44 & 1.34 \\
\hline & ${ }^{18} \mathrm{~F}-\mathrm{DPA} 714$ & 1.33 & 1.34 & 1.31 & 1.23 \\
\hline \multirow[t]{2}{*}{ HV SD } & ${ }^{11} \mathrm{C}-\mathrm{PBR} 28$ & $0.21(0.16)$ & $0.11(0.08)$ & $0.13(0.11)$ & $0.07(0.06)$ \\
\hline & ${ }^{18} \mathrm{~F}-\mathrm{DPA} 714$ & $0.22(0.21)$ & $0.09(0.07)$ & $0.22(0.22)$ & $0.14(0.13)$ \\
\hline \multirow[t]{2}{*}{ ALS SD } & ${ }^{11} \mathrm{C}-\mathrm{PBR} 28$ & $0.18(0.11)$ & $0.12(0.08)$ & $0.14(0.10)$ & $0.13(0.10)$ \\
\hline & ${ }^{18}$ F-DPA714 & $0.16(0.12)$ & $0.06(0.04)$ & $0.12(0.09)$ & $0.09(0.08)$ \\
\hline \multirow[t]{2}{*}{ Effect size } & ${ }^{11} \mathrm{C}-\mathrm{PBR} 28$ & 1.17 & 0.10 & 1.41 & 1.76 \\
\hline & ${ }^{18}$ F-DPA714 & 1.34 & 1.27 & 1.32 & 1.20 \\
\hline
\end{tabular}

in ${ }^{18}$ F-DPA714 PET analyses of $\mathrm{SUVR}_{40-60}$ was WB without ventricles, providing results that were even more robust than analyses using $\mathrm{V}_{\mathrm{T}}$. Therefore, we suggest using WB without ventricles as the pseudo reference region for future ${ }^{18} \mathrm{~F}$-DPA714 studies on $\mathrm{ALS}$, in line with previous ${ }^{11} \mathrm{C}-\mathrm{PBR} 28$ studies in which $\mathrm{WB}$ normalization was applied $(7-9,13)$. No significant differences in absolute $\mathrm{V}_{\mathrm{T}}$ were observed between ALS subjects and HVs, probably because the Belgian HVs were not perfectly matched for age and genotype to the Belgian ALS subjects and the sample size was small; these are limitations of this study.

This study was an initial effort to combine and compare data acquired from 2 separate cohorts of ALS participants with separate tracers and 2 different scanners, using a fully automated analysis pipeline and pseudo reference region analysis technique. To the best of our knowledge, there have been no prior direct comparison studies of ${ }^{18} \mathrm{~F}$-DPA714 and ${ }^{11} \mathrm{C}$-PBR28 PET scans. We demonstrated comparable localization and uptake changes in clinically meaningful regions in ALS subjects. The full factorial analysis demonstrated that the variance of the group effect could be explained only by the bilateral glial activation in the primary motor cortices. This finding illustrates the robustness of the neuroinflammation pattern that we observed across both tracers. Therefore, our study provides early clues on the feasibility of conducting future multicenter and multitracer TSPO brain PET studies in ALS.

Our preliminary results suggest that ${ }^{18} \mathrm{~F}$-DPA714 may have a higher sensitivity than ${ }^{11} \mathrm{C}$-PBR28, as we observed higher-uptake signal changes and a wider distribution of high-uptake voxels within the primary motor cortex region of interest. However, the effect sizes and bilateral variability in the primary motor cortices of HVs were comparable. Because one limitation of this preliminary study is the rather heterogeneous participant characteristics-in terms of clinical phenotype and degree of upper motor neuron dysfunctionthis heterogeneity seems the most likely explanation of the more extensive pattern of glial activation using ${ }^{18} \mathrm{~F}-\mathrm{DPA} 714$. Also, disease duration and revised ALS functional rating scale significantly differed between the ALS groups, with the Belgian ALS group scanned earlier in the course of their disease.

The relatively small sample size is another limitation of this study. Nevertheless, this pilot study was intended to function as a proof of principle for pooling data from 2 TSPO tracers. In the future, we aim to increase our number of participants for pattern characterization and to include participants who have different disease stages.

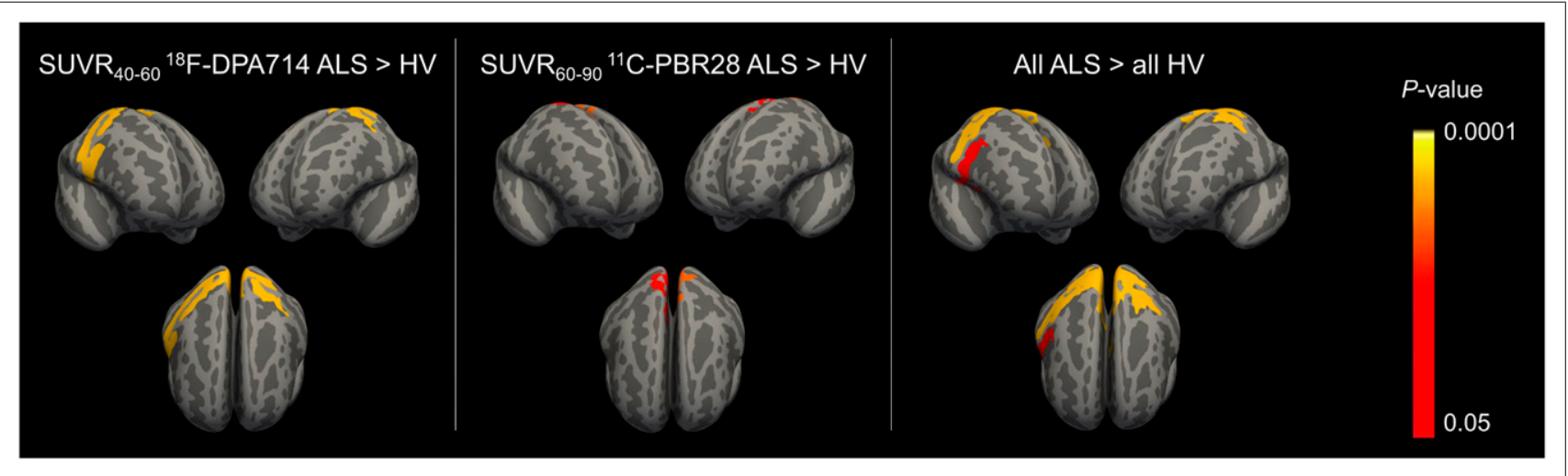

FIGURE 3. Surface-based analysis between participants with ALS and HVs for ${ }^{18} \mathrm{~F}-\mathrm{DPA} 714 \mathrm{SUVR}_{40-60},{ }^{11} \mathrm{C}-\mathrm{PBR} 28$ SUVR $60-90$, and both tracers $\left({ }^{18} \mathrm{~F}-\mathrm{DPA} 714 \mathrm{SUVR}_{40-60}\right.$ combined with $\left.{ }^{11} \mathrm{C}-\mathrm{PBR} 28 \mathrm{SUVR}_{60-90}\right)$ using WB without ventricles as pseudo reference region. 
Additionally, we could not perform a head-to-head comparison between ${ }^{18} \mathrm{~F}$-DPA714 and ${ }^{11} \mathrm{C}$-PBR28 in the same participants to assess intrasubject variability across ligands. This head-to-head comparison will be needed to determine which TSPO tracer has higher sensitivity in detecting the specific distribution patterns in ALS - and which has better intrasubject versus intersubject variance in tracer uptake intensity - for future quantitative TSPO PET studies. Our future studies will be designed to evaluate if ${ }^{18} \mathrm{~F}$-DPA714 has higher sensitivity (as seen in this study) because of tracer effect or because of biologic differences in disease mechanisms in different ALS participants.

Currently, all TSPO PET studies require genotyping of the rs6971 polymorphism to determine TSPO binding affinity and to exclude low-affinity binders ( $\sim 5 \%-10 \%$ of the population) because low-affinity binders have a lower specific signal, which may affect PET data analysis (19). In our study, the ${ }^{18} \mathrm{~F}$-DPA714 SUVR in the 1 low-affinity binder was comparable to that in the medium- and high-affinity binders. Although this single finding is not sufficient to answer whether TSPO binding affinity significantly influences ${ }^{18} \mathrm{~F}$ DPA714 PET SUVR results, it warrants future studies. If the SUVR analysis of ${ }^{18} \mathrm{~F}$-DPA714 binding in the left and right primary motor cortices can robustly detect differences between ALS participants and other groups regardless of the TSPO binding affinity genotype of the participant, this ability will enable increased recruitment of ALS participants in future TSPO PET studies and reduce the costs associated with TSPO genotyping.

This study demonstrated the feasibility of combining multicenter PET data acquired on different scanners, with different PET cameras, using 2 different TSPO radioligands. PVC played a nonnegligible role in this finding, because the ability of PVC to partially compensate for differences in resolution is critical in neurodegenerative diseases with substantial atrophy. Moreover, this study showed that - because of the consistent neuroinflammation pattern observed for both tracers- the pattern of neuroinflammation in ALS can be visualized more perspicuously when single-site data are pooled into larger multicenter cohorts. This finding illustrates the feasibility of combining multicenter, multitracer, and multiscanner data for qualitative assessment of the presence or absence of brain neuroinflammation in ALS participants. A similar approach has been implemented for amyloid PET scans in the Centiloid Project. In this project, a standard data analysis approach has been developed to enhance data comparison across different tracers and centers and, thus, facilitate multicenter trials $(20,21)$. Likewise, multicenter TSPO PET can serve to enrich cohorts for future neuroinflammation biomarker studies in ALS. Until now, trials of anti-inflammatory drugs in ALS have shown no or only very moderate effects on ALS progression (22). Future trials of anti-inflammatory or immunomodulatory drugs in ALS may be more successful if they are tested on a cohort enriched for participants with a TSPO PET-demonstrated inflammatory component of disease.

One of the main challenges in ALS drug development is the lack of biomarkers that can be used in proof-of-concept phase 2 clinical trials. As a result, phase 2 clinical trials in ALS use clinical endpoints that require very large sample sizes. This study was a critical first step toward demonstrating that pooling of multicenter TSPO PET data improves power for future phase 2 clinical trials in ALS. Drug trials may use TSPO PET as a stratifying biomarker for trial entry or use TSPO PET as a pharmacodynamic biomarker of drug effect. In summary, this study introduced the potential use of TSPO PET as a measure of the biologic efficacy of investigational drugs for ALS across various geographic locations and TSPO PET tracer capabilities at multiple centers. In this regard, we believe that our findings will accelerate therapeutic development for people with ALS.

\section{CONCLUSION}

The pseudo reference region approach can be used for both ${ }^{18} \mathrm{~F}$ DPA714 and ${ }^{11} \mathrm{C}-\mathrm{PBR} 28$ in ALS and produces results similar to or even more robust than those obtained with the gold standard, $\mathrm{V}_{\mathrm{T}}$, using WB without ventricles as an optimal pseudo reference region. Moreover, this approach allows data pooling of 2 second-generation TSPO radioligands, ${ }^{11} \mathrm{C}-\mathrm{PBR} 28$ and ${ }^{18} \mathrm{~F}-\mathrm{DPA} 714$, facilitating multicenter trials.

\section{DISCLOSURE}

Koen Van Laere and Philip Van Damme are senior clinical investigators of the Fund for Scientific Research, Flanders, Belgium (FWO). Donatienne Van Weehaeghe is a $\mathrm{PhD}$ fellow of the FWO $(1179620 N)$. Philip Van Damme is supported through the E. von Behring Chair for Neuromuscular and Neurodegenerative Disorders, the ALS Liga Belgie and KU Leuven funds "Een Hart voor ALS" and "Laeversfonds voor ALS Onderzoek," and the "Valéry Perrier race against ALS" fund. Nazem Atassi is supported through NIHK23-NS083715, the Muscular Dystrophy Association, the ALS Association, and ALS Finding a Cure. The postdoc position of Georg Schramm is funded by NIH grant 1P41EB017183-01A1. No other potential conflict of interest relevant to this article was reported.

\section{ACKNOWLEDGMENTS}

We thank Kwinten Porters and Jef Van Loock for their contributions to the scanning and data handling, and we thank the PET radiopharmacy team and the medical physics team of UZ Leuven for their skilled contributions. We also thank the radiopharmacy team, the nuclear medicine technologists, and the nurses of the A.A. Martinos Center for radiotracer synthesis and assistance with PET/MRI.

\section{KEY POINTS}

QUESTION: First, can we apply to another second-generation TSPO radioligand the pseudo reference region approach currently used for ${ }^{11} \mathrm{C}-\mathrm{PBR} 28$ for noninvasive simplified PET imaging of ${ }_{18}^{18} \mathrm{~F}-\mathrm{DPA} 714$ ? Second, can we pool multicenter ${ }^{18} \mathrm{~F}-\mathrm{DPA} 714$ and ${ }^{11} \mathrm{C}$-PBR28 PET scans from ALS subjects and HVs using a pseudo reference region approach?

FINDINGS: We demonstrated that combining multicenter data using TSPO radioligands to visualize neuroinflammation is feasible. WB without ventricles yielded the highest sensitivity as a pseudo reference region for ${ }^{18} \mathrm{~F}-\mathrm{DPA} 714$. Data pooling across tracers and study centers resulted in higher sensitivity to detect widespread increased glial activation, with signal intensity differences of about $20 \%$ in the left and right primary motor cortices in ALS subjects compared with HVs.

IMPLICATIONS FOR PATIENT CARE: The data from this study can facilitate conducting multicenter TSPO studies, which are essential to improve statistical power for disease detection, patient classification, and potential therapy monitoring. 


\section{REFERENCES}

1. Hardiman O, Al-Chalabi A, Chio A, et al. Amyotrophic lateral sclerosis. Nat Rev Dis Primers. 2017;3:17085.

2. Liu J, Wang F. Role of neuroinflammation in amyotrophic lateral sclerosis: cellular mechanisms and therapeutic implications. Front Immunol. 2017;8:1005.

3. Beers DR, Appel SH. Immune dysregulation in amyotrophic lateral sclerosis: mechanisms and emerging therapies. Lancet Neurol. 2019;18:211-220.

4. Werry EL, Bright FM, Piguet O, et al. Recent developments in TSPO PET imaging as a biomarker of neuroinflammation in neurodegenerative disorders. Int J Mol Sci. 2019;20:3161.

5. Owen DR, Yeo AJ, Gunn RN, et al. An 18-kDa translocator protein (TSPO) polymorphism explains differences in binding affinity of the PET radioligand PBR28. J Cereb Blood Flow Metab. 2012;32:1-5.

6. Alam MM, Lee J, Lee SY. Recent progress in the development of TSPO PET ligands for neuroinflammation imaging in neurological diseases. $\mathrm{Nucl} \mathrm{Med} \mathrm{Mol}$ Imaging. 2017;51:283-296.

7. Zürcher NR, Loggia ML, Lawson R, et al. Increased in vivo glial activation in patients with amyotrophic lateral sclerosis: assessed with $\left[{ }^{11} \mathrm{C}\right]-\mathrm{PBR} 28$. Neuroimage Clin. 2015;7:409-414.

8. Alshikho MJ, Zurcher NR, Loggia ML, et al. Glial activation colocalizes with structural abnormalities in amyotrophic lateral sclerosis. Neurology. 2016;87:2554-2561.

9. Alshikho MJ, Zurcher NR, Loggia ML, et al. Integrated magnetic resonance imaging and $\left[{ }^{11} \mathrm{C}\right]-\mathrm{PBR} 28$ positron emission tomographic imaging in amyotrophic lateral sclerosis. Ann Neurol. 2018;83:1186-1197.

10. Corcia P, Tauber C, Vercoullie J, et al. Molecular imaging of microglial activation in amyotrophic lateral sclerosis. PLoS One. 2012;7:e52941.

11. Ratai EM, Alshikho MJ, Zurcher NR, et al. Integrated imaging of $\left[{ }^{11} \mathrm{C}\right]-\mathrm{PBR} 28$ PET, MR diffusion and magnetic resonance spectroscopy ${ }^{1} \mathrm{H}-\mathrm{MRS}$ in amyotrophic lateral sclerosis. Neuroimage Clin. 2018;20:357-364.

12. Van Weehaeghe D, Van Schoor E, De Vocht J, et al. TSPO versus P2X7 as a target for neuroinflammation: an in vitro and in vivo study. J Nucl Med. 2020;61: 604-607.
13. Albrecht DS, Normandin MD, Shcherbinin S, et al. Pseudoreference regions for glial imaging with ${ }^{11} \mathrm{C}-\mathrm{PBR} 28$ : investigation in 2 clinical cohorts. J Nucl Med. 2018;59:107-114.

14. Lyoo CH, Ikawa M, Liow JS, et al. Cerebellum can serve as a pseudo-reference region in Alzheimer disease to detect neuroinflammation measured with PET radioligand binding to translocator protein. J Nucl Med. 2015;56:701706.

15. Rezaei A, Schramm G, Van Laere K, Nuyts J. Estimation of crystal timing properties and efficiencies for the improvement of (joint) maximum-likelihood reconstructions in TOF-PET. IEEE Trans Med Imaging. 2020;39:952-963.

16. Schramm G, Koole M, Willekens SMA, et al. Regional accuracy of ZTE-based attenuation correction in static $\left[{ }^{18} \mathrm{~F}\right] \mathrm{FDG}$ and dynamic $\left[{ }^{18} \mathrm{~F}\right] \mathrm{PE} 2 \mathrm{I}$ brain PET/MR. Front Phys. December 6, 2019 [Epub ahead of print].

17. Lavisse S, Garcia-Lorenzo D, Peyronneau MA, et al. Optimized quantification of translocator protein radioligand ${ }^{18} \mathrm{~F}$-DPA-714 uptake in the brain of genotyped healthy volunteers. J Nucl Med. 2015;56:1048-1054.

18. Izquierdo-Garcia D, Hansen AE, Forster S, et al. An SPM8-based approach for attenuation correction combining segmentation and nonrigid template formation: application to simultaneous PET/MR brain imaging. J Nucl Med. 2014;55: 1825-1830.

19. Owen DR, Howell OW, Tang SP, et al. Two binding sites for $\left[{ }^{3} \mathrm{H}\right] \mathrm{PBR} 28$ in human brain: implications for TSPO PET imaging of neuroinflammation. J Cereb Blood Flow Metab. 2010;30:1608-1618.

20. Su Y, Flores S, Wang G, et al. Comparison of Pittsburgh compound B and florbetapir in cross-sectional and longitudinal studies. Alzheimers Dement (Amst). 2019;11:180-190.

21. Klunk WE, Koeppe RA, Price JC, et al. The centiloid project: standardizing quantitative amyloid plaque estimation by PET. Alzheimers Dement. 2015;11: 1-15 e11-14.

22. Crisafulli SG, Brajkovic S, Cipolat Mis MS, Parente V, Corti S. Therapeutic strategies under development targeting inflammatory mechanisms in amyotrophic lateral sclerosis. Mol Neurobiol. 2018;55:2789-2813. 